Published online 2017 April 13.

Abstract

\title{
New Varicosis Treatments and New Keynots
}

\author{
Kambiz Faridmarandi, ${ }^{1, *}$ and Hossein Hemmati ${ }^{2}$ \\ ${ }^{1}$ Marlic Radiology Clinic \\ ${ }^{2}$ GUMS \\ "Corresponding author: Kambiz Faridmarandi, Marlic Radiology Clinic. E-mail: Kambizfaridmarandi@yahoo.com
}

Received 2016 December 21; Accepted 2017 February 08.

\begin{abstract}
Lower extremity venous varicosis is a very prevalent disease. Approximately 15 - 25 percent of the general population suffer from some degree of this disease. In recent years many new and innovative treatments have been developed for the disease and nowadays customary treatments ( saphenous strapping and phlebotomy) are nearly obsolete. These new treatments include Laser ablation, Radio frequency ablation, Glue, sclerotherapy, foam sclerotherapy and reveloutionaized treatment of venous varicosis. These new technologies require new data that must be mentioned in duplex reports. These keynots determine which treatment is suitable for the patient and which one is not. The goal of this article is about to explain the technical aspects of the treatments and new informations that must be added to duplex reports.
\end{abstract}

This is an abstract presented in the 33rd Iranian congress of radiology (ICR) and the 15th congress of Iranian radiographic science association (IRSA). 